\title{
Effective detection of weak terahertz pulses in electro-optic sampling at kilohertz repetition rate
}

\author{
Philipp Krauspe, Natalie BanerJi, and Julien Réhault* (1) \\ Universität Bern, Department of Chemistry and Biochemistry, Freiestrasse 3, 3012 Bern, Switzerland \\ ${ }^{*}$ Corresponding author: julien.rehault@dcb.unibe.ch
}

Received 26 September 2019; revised 19 November 2019; accepted 19 November 2019; posted 22 November 2019 (Doc. ID 378839 ); published 17 December 2019

\begin{abstract}
The standard terahertz ( $\mathrm{THz})$ detection mechanism known as electro-optic sampling can be improved in sensitivity by biasing the polarization of the sampling field. In this work, we show theoretically and experimentally how weak signals can be amplified without inducing distortions. Our study identifies the influence of THz field strength, the polarization quality, and biasing amplitude on signal amplification and distortion. Here we present a distortionfree amplification of a factor of $\mathbf{2 8}$ while at the same time reducing the measurement time significantly. (c) 2019 Optical Society of America
\end{abstract}

https://doi.org/10.1364/JOSAB.37.000127

\section{INTRODUCTION}

Measuring terahertz $(\mathrm{THz})$ fields in the picosecond range requires the mixing of a $\mathrm{THz}$ pulse with a sampling light pulse of much shorter duration. One method to measure such a pulse is electro-optic sampling (EOS). EOS is based on the optical Kerr effect in the limit of the THz electric field oscillating slowly compared with the gating light pulse duration. Concise and indepth description of the process has been given in the frequency domain [1] from the optical intensities measured in the process [2], and starting from the polarization components [3]. One typical use of this detection scheme is employed in optical pump $\mathrm{THz}$ probe studies, where electronic properties of materials are optically probed, making it an especially powerful tool in optoelectronics research. For many studied materials, the lifetimes of optical excitations reach microseconds; thus, amplified Ti:Sa laser systems with low repetition rates in the kilohertz $(\mathrm{kHz})$ range are often necessary in materials science. Compared with $\mathrm{MHz}$ systems, acquiring enough laser shots to reach an adequate signal-to-noise ratio significantly increases measurement time, motivating the improvement of the detection scheme. Several methods were previously employed, all relying on the excess of sampling pulse intensity available when using $\mathrm{kHz}$ repetition rate systems, where the damage thresholds of the optics or detectors in use are the limiting factors. Typically, the sampling pulse intensity is attenuated to avoid saturation of the detector. As EOS basically is a translation of field strength into change of polarization, it is possible to take advantage of the excess energy to improve detection sensitivity with careful manipulation of the polarization in the detection process. Previous studies achieved that by biasing the input polarization with an optical retarder before [4] and/or after the optical mixing within the detection crystal $[3,5]$. In a comparable scheme, a series of wedges was used to control the polarization [6]. The bias can also be introduced using nearly crossed polarizers [3]. Unbalanced detection, which is employed in $\mathrm{THz}$ spectrometers based on asynchronous optical sampling (ASOPS) [7], is also closely related to these. Within these schemes, distortions of the measured $\mathrm{THz}$ pulse have been identified as a key limiting factor. Distortion-free amplification techniques have been reported, but the usability is limited since either two measurements are needed to reconstruct the true waveform [5] or they are not applicable to detect weak THz fields [4]. In this paper, we show how the strength of the $\mathrm{THz}$ field itself is a key parameter to limit amplification and distortion as hypothesized before [6]. Additionally, we show how distortion-free amplification is possible at a small $\mathrm{THz}$ field, allowing us to detect weak signals with increased sensitivity. To this purpose, we used a simple scheme presented before by Johnson et al. [5] and Brunner et al. [3] and apply the Mueller formalism to propagate the polarization state through the detection. This allows us (i) to account for the imperfect polarization of the sampling pulse (also defined as gate pulse in this work) and (ii) to understand the origin and the link between distortions, THz strength, and amplification in detail. We present the experimental setup followed by its theoretical description and demonstrate its applicability in the detection of weak THz fields.

\section{METHODS}

\section{A. Setup for Detectivity Enhanced Electro-Optic Sampling of THz Pulses in the kHz Range}

Figure 1 shows the typical setup for EOS with bias as published by Johnson et al. [5]. We take our $1 \mathrm{kHz}, 35 \mathrm{fs}$ light pulse at $800 \mathrm{~nm}$ and split it into two beams for the $\mathrm{THz}$ generation 


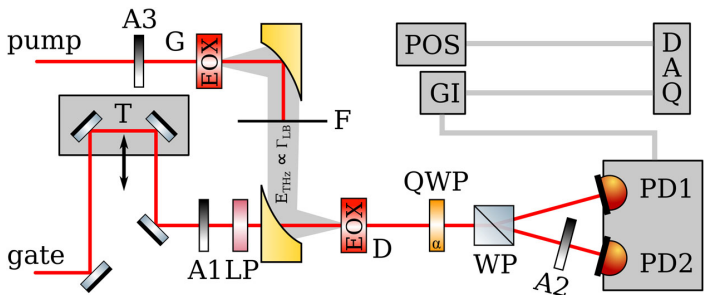

Fig. 1. Experimental setup: generation crystal, G; detection crystal, D; quarter-wave plate, QWP; polarizing beam splitter, WP; two balanced photodiodes, PD1, PD2; variable attenuators, A1, A2, A3; linear polarizer, LP; PTFE filter, F; gated integrator, GI; position encoding, POS; directly from linear translation stage, T; data acquisition, DAQ.

(pump) and detection (gate). The pump generates the $\mathrm{THz}$ pulse in the generation crystal $(G)$ via optical rectification in a $1 \mathrm{~mm}$ thick ZnTe crystal (EOX). The THz pulse is imaged with two off-axis parabolic mirrors into our detection crystal (D). A PTFE filter (F) transmits the THz pulse and blocks the residual $800 \mathrm{~nm}$ light. The linearly polarized gating beam (through LP: colorPol VIS-IR polarizer, CODIXX) is spatially and temporally overlapped with the $\mathrm{THz}$ pulse. The collinear spatial and directional overlap is achieved by drilling a hole into our focusing parabola and directing the gate through it. Temporal overlap is achieved by modifying the length of the gate's optical pathway with a translation stage $(T)$. Scanning the gate arrival time over the $\mathrm{THz}$ waveform reconstructs the electric field by measuring its effect on the birefringence on the detection crystal $[1,2]$. This $\mathrm{THz}$-induced birefringence $\left(\Gamma_{\mathrm{LB}}\right)$ is then analyzed by a series of quarter-wave plates (QWPs), Wollaston prisms (WPs), and balanced photodiodes (PD1, PD2).

Classically, the birefringent $\mathrm{ZnTe}$ detection crystal is oriented along the polarization of the incoming detection beam and the wave plate at $45^{\circ}$, such that ideally perfectly circular polarized light is split 50:50 from the WP [2]. Without $\mathrm{THz}$, the diodes are balanced so the signal $S=I_{1}-I_{2}=0$, with $I_{1}, I_{2}$ being the intensities on PD1 and PD2, respectively. With THz, that difference leads to a finite value of $S$.

In our implementation, $(\mathrm{T})$ is a magnetic delay stage (V-508 PIMag, PI Instruments), with which fast scanning is employed to evenly distribute slow pulse-to-pulse fluctuations of the laser system over the whole waveform [8]. Here, rapid scanning is achieved by simultaneously reading the voltage from our gated integrator (GI) connected to the balanced PDs and the position of the stage, so that the position is recorded for every laser shot. We derive the position by externally sensing several contacts in the cable connecting stage and controller PI PIMAG C981, while pulling the voltage level to transistor-transistor logic (TTL) levels to read the position with the counter on a digital input.

To take special care about the polarization state within the detection, we sequentially add optics into the system. First, we ensure that LP exactly aligns with one output port of the WP such that all intensity is on PD2. Then, we introduce the detection crystal EOX-D along one of its crystal axes to ensure minimal residual birefringence. Lastly, the QWP is added in a motorized rotational mount (K10CR1, Thorlabs) to reproducibly set angles with high precision.

With A2 set to no attenuation, the overall used intensity is set at $\mathrm{A} 1$ to ensure linear behavior on the diodes PD1 and PD2. To amplify the measured signal, we bias the gate by setting the wave plate at an angle of $\alpha \neq 45^{\circ}$ and attenuate (A2) after the WP to reduce $I_{2}$ and thus rebalance the intensities on the detector, as described in Refs. [3,5]. For a given damage threshold of EOX$\mathrm{D}$, one can increase the input intensity of the gating beam and still be at balanced detection. In an extreme case, one removes the QWP such that $I_{2}=I_{0}$ and $I_{1}=0$ without THz. This resembles the cross-polarizer measurement scheme [9]. With strong $\mathrm{THz}$ pulses and a sufficiently clean polarization state, this leads to the detection of purely positive signals (homodyne) corresponding to strong distortions reported in Ref. [5]. For a set of wave plate angles, we measure the $\mathrm{THz}$ waveform keeping the intensity constant on the diodes.

\section{B. Theoretical Description of Detectivity Enhancement in Electro-Optic Sampling}

The effect of the THz field in the detection crystal (EOX-D) is to create a linear birefringence $\Gamma_{\mathrm{LB}}$ at $45^{\circ}$ [10], proportional to the $\mathrm{THz}$ electric field strength. This way, it modulates the initially horizontally polarized gating beam. With QWP set at $\alpha$ and the intensity on the diodes kept constant with $\mathrm{A} 1$ and $\mathrm{A} 2$, propagation of the polarization state through the optical elements as a Stokes vector in Mueller formalism yields the signal $S$. With perfect polarizers $(r=0, r$ being the extinction ratio of the polarizing optics) and in the limit of small $\Gamma_{\mathrm{LB}}\left(\Gamma_{\mathrm{LB}} \ll 45^{\circ}\right)$, we obtain

$$
S\left(\alpha, \Gamma_{\mathrm{LB}}\right)=\frac{\Gamma_{\mathrm{LB}}}{\sin (2 \alpha)}+\frac{1}{2} \cot (2 \alpha)^{2} \cdot \Gamma_{\mathrm{LB}}^{2} .
$$

The first term, linear in $\Gamma_{\mathrm{LB}}$, is referred to as the heterodyne (or self-heterodyne) signal. It is linear in $\Gamma_{\mathrm{LB}}$ (THz field strength) and thus induces no distortion. The second term is the so-called homodyne signal. The homodyne term is quadratic in $\Gamma_{\mathrm{LB}}$ and is responsible for the distortions. At small angles $\alpha$, the signal diverges because this equation ignores finite gating light intensity at the input. Subtracting two measurements at $\pm \alpha$ doubles $S\left(\alpha, \Gamma_{\mathrm{LB}}\right)$ while eliminating the homodyne component leading to an undistorted signal $[3,5]$. On the contrary, adding them yields twice the purely positive homodyne signal,

$$
\begin{aligned}
& S\left(\alpha, \Gamma_{\mathrm{LB}}\right)-S\left(-\alpha, \Gamma_{\mathrm{LB}}\right)=\frac{2 \Gamma_{\mathrm{LB}}}{\sin (2 \alpha)}, \quad \text { and } \\
& S\left(\alpha, \Gamma_{\mathrm{LB}}\right)+S\left(-\alpha, \Gamma_{\mathrm{LB}}\right)=\cot (2 \alpha)^{2} \cdot \Gamma_{\mathrm{LB}}^{2} .
\end{aligned}
$$

In the Mueller formalism (see Appendix A), we account for imperfections in the polarization $(r>0)$. In theory, the signal is as follows:

$$
S_{r}\left(\alpha, \Gamma_{\mathrm{LB}}\right)=\frac{\left.\cos (2 a)^{2}\left(-2+4 r^{2}-2 r^{4}\right)+\left(2-4 r^{2}+2 r^{4}\right)^{2} \cos \left(\Gamma_{\mathrm{LB}}\right)+\left(-2+4 r^{2}-2 r^{4}\right) \sin (2 \alpha) \cdot \sin \Gamma_{\mathrm{LB}}\right]}{-(1+r)^{4}+(1-r)^{4} \cos (2 \alpha)^{4}} .
$$




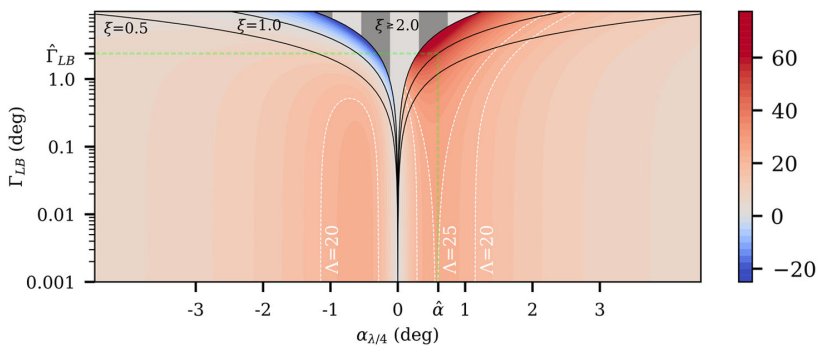

Fig. 2. $2 \mathrm{D}$ representation of amplification $\Lambda_{r}$ [Eq. (2)] depending on $\Gamma_{\mathrm{LB}}$ and $\alpha$ at $r=10^{-4}$. Black lines depict distortion $\xi_{\mathrm{r}}\left(\alpha, \Gamma_{\mathrm{LB}}\right)=0.5,1.0$ and $\xi_{\mathrm{r}}\left(\alpha, \Gamma_{\mathrm{LB}}\right) \geq 2.0$, respectively [Eq. (3)]. In the shaded region with $\xi_{r} \geq 2.0$, distortions dominate the signal. $\hat{\Gamma}_{\mathrm{LB}}=2.37^{\circ}$ corresponds to the peak field strength of the pulses used in the measurements presented in this work. $\hat{\alpha}$ depicts the optimized angle in this case. The green dashed line indicates both in the graph. White dashed lines depict boundaries of $\Lambda_{r}\left(\alpha, \Gamma_{\mathrm{LB}}\right)=20$ and $\Lambda_{r}\left(\alpha, \Gamma_{\mathrm{LB}}\right)=25$, respectively.

We define the amplification $\Lambda$ at any $\pm \alpha$ relative to the signal measured at $\alpha= \pm 45^{\circ}$, respectively,

$$
\Lambda_{\mathrm{r}}\left(\alpha, \Gamma_{\mathrm{LB}}\right)=\frac{S_{r}\left(\Gamma_{\mathrm{LB}}, \pm \alpha\right)}{S_{r}\left(\Gamma_{\mathrm{LB}}, \alpha= \pm 45^{\circ}\right)} .
$$

The distortion $\xi$ is defined as the ratio of homodyne (distorted) to the self-heterodyne (undistorted) signal,

$$
\xi_{\mathrm{r}}\left(\alpha, \Gamma_{\mathrm{LB}}\right)=\left|\frac{S_{r}\left(\alpha, \Gamma_{\mathrm{LB}}\right)+S_{r}\left(-\alpha, \Gamma_{\mathrm{LB}}\right)}{S_{r}\left(\alpha, \Gamma_{\mathrm{LB}}\right)-S_{r}\left(-\alpha, \Gamma_{\mathrm{LB}}\right)}\right| .
$$

In this way, the case $\xi=0$ contains no homodyne signal resulting in no distortions, while for $\xi>0$, the influence of the homodyne signal distorts the measured waveform. $\xi$ increases with $\Gamma_{\mathrm{LB}}$; however, at small $\Gamma_{\mathrm{LB}}$, we already find significant amplification with only negligible distortions, which is the focus of this study. To validate this theory experimentally, measurements are taken up to a maximally applied field (peak of the $\mathrm{THz}$ waveform) of $\hat{\Gamma}_{\mathrm{LB}}=2.37^{\circ}$ (in the distortion regime).

In Fig. 2, we display a 2D map of $\Lambda_{\mathrm{r}}$ in function of $\alpha$ and positive $\Gamma_{\mathrm{LB}}$, with $r$ set to $r=10^{-4}$, which is close to our experimental conditions. We show the area with small $\alpha$ to highlight the regime where significant amplification $\Lambda_{\mathrm{r}}$ is possible. $\Lambda_{r}$ is color coded from blue to red going from negative to positive amplification. Negative amplification is present when the homodyne contribution to the signal takes over. The black line at $\xi_{\mathrm{r}}=1.0$ for $\alpha<0$ marks this case where $\Lambda_{\mathrm{r}}=0$, and, thus, the sign flips.

Distortions increase with higher $\Gamma_{\mathrm{LB}}$ and smaller $\alpha$. The solid black lines depict signals with distortions of $\xi_{\mathrm{r}}=0.5$, $\xi_{\mathrm{r}}=1.0$, and $\xi_{\mathrm{r}} \geq 2.0$, respectively. The area for $\xi_{\mathrm{r}} \geq 2.0$, where the homodyne signal is twice the heterodyne contribution, is grayed out for illustrative purposes because the amplification diverges at large $\Gamma_{\mathrm{LB}}$. Contour lines of $\Lambda_{\mathrm{r}}=20$ and $\Lambda_{\mathrm{r}}=25$ are shown as white dashed lines. The area below $\xi_{\mathrm{r}}=0.5$ is where distortions are small, such that areas of strong amplification without significant distortions can readily be identified.

We observe that when $\Gamma_{\mathrm{LB}}$ is small, we can use small $\alpha$ without distorting the recovered $\mathrm{THz}$ field significantly.
Furthermore, there is an optimal angle at $\hat{\alpha}=\sqrt{r}$ for which the strongest amplification is achieved while distortions can be neglected (if $\Gamma_{\mathrm{LB}}$ is sufficiently small). Then, the amplification at this angle converges to $\hat{\Lambda}=1 /(4 \sqrt{r})$ when $\Gamma_{\mathrm{LB}} \rightarrow 0$. In the particular case of this figure with $r=10^{-4}$, the angle is $\hat{\alpha}=0.01\left(0.6^{\circ}\right)$ and leads to an amplification of $\hat{\Lambda}=25$. Because the amplification only approaches $\hat{\Lambda}=25$, we do not find exactly the same for both $\pm \alpha$. This is due to the small homodyne contribution where the sign of the effect depends on the relative sign between $\alpha$ and $\Gamma_{\mathrm{LB}}$. Once the introduced optical bias in $\alpha$ does not compete with the field strength $\Gamma_{\mathrm{LB}}$, we are in the limit of distortion-free amplification of weak fields.

\section{RESULTS AND DISCUSSION}

In Fig. 3(a), we show the THz electric field transients at a representative selection of angles $\alpha$ of the QWP. The subtracted signals of the respective $\pm \alpha$ counterparts are shown in Fig. 3(b), showing nondistorted signals following [5]. The inset in Fig. 3(b) shows these same waveforms normalized to demonstrate that fact. We find a distortion-free amplification factor of $\sim 24$, comparing the subtracted signals at $0.7^{\circ}$ with a single measurement at $45^{\circ}$, which closely agrees with the predictions made in the previous section. We present two ways of avoiding the second measurement to reconstruct the waveform. First, if the applied field is weak compared with the optical bias, and the single measurement with $+\alpha$ will be distortion-free, meaning the measurement at $-\alpha$ will have the opposite sign such that the subtracted signals will just be 2 times the measurement at $+\alpha$. Second, the applied field can be reconstructed by fitting Eq. (1) to the distorted measurement data as shown in Fig. 4(b). For strongly distorted signals where the sign flips $\left(\Gamma_{\mathrm{LB}} \geq \alpha\right)$, the mapping with $S_{r}\left(\alpha, \Gamma_{\mathrm{LB}}\right)$ is not bijective anymore; thus, fitting requires knowledge of the original signals' shape. From
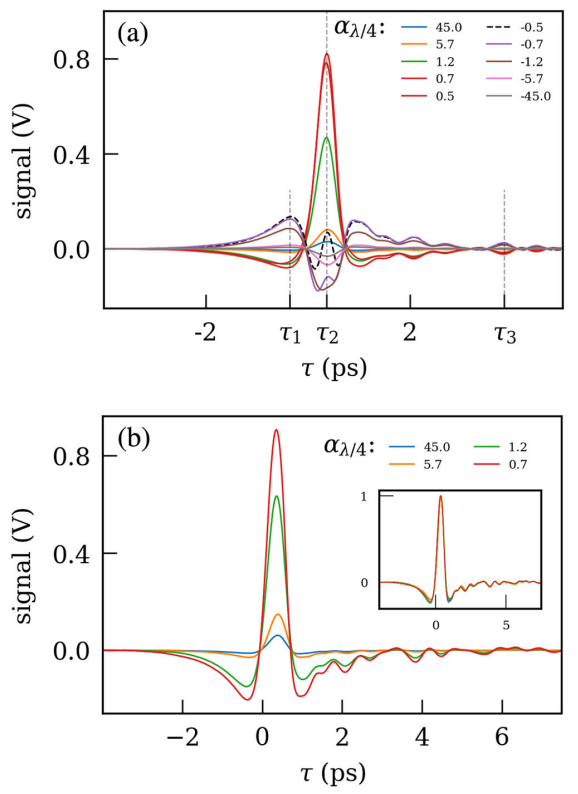

Fig. 3. (a) Collection of the measured signals at constant power on the diodes with different angles $\alpha$ of the quarter-wave plate. (b) Subtracted signals of corresponding quarter-wave plate angles $( \pm \alpha)$ with their respective normalized signals in the inset. 

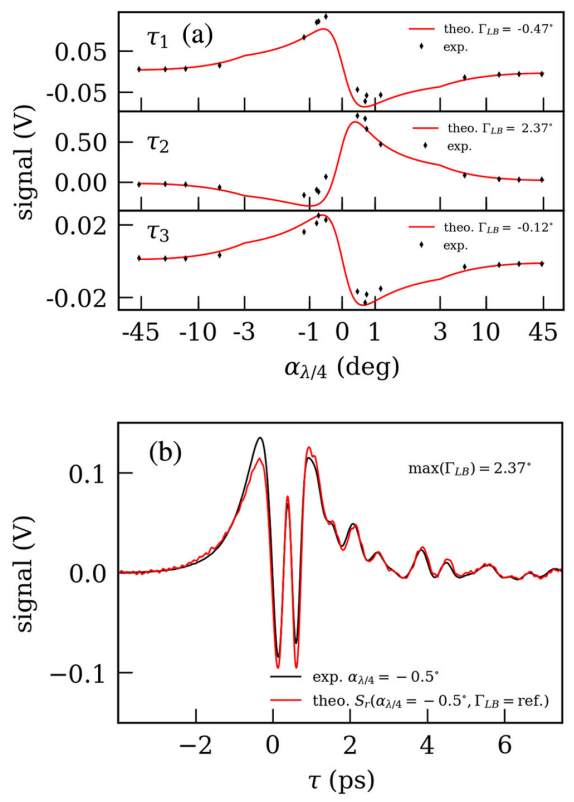

Fig. 4. (a) $S_{r}\left(\Gamma_{\mathrm{LB}}, \alpha\right)$ and measured signal for three $\Gamma_{\mathrm{LB}}$ selected from $\tau$ in Fig. 3(a). (b) Measurement and calculated signal for the whole waveform at a small angle $\alpha=-0.5^{\circ}$.

Fig. 3(a), it becomes evident how small angles lead to higher measured signals, including an asymmetry at small angles that appears strongest at the peak of the $\mathrm{THz}$ field. We determine $\Gamma_{\mathrm{LB}}$ experimentally by compensating the $\mathrm{THz}$ signal rotating the QWP for each $\tau_{i}$ depicted in Fig. 3(a). The wave plate angle is directly related to $\Gamma_{\mathrm{LB}}$. The $\tau_{i}$ corresponds to a minimum field at $\tau_{1}$, the field maximum at $\tau_{2}$, and a small nonvanishing field at $\tau_{3}$.

Figure 4(a) shows the signal at the 3 times $\tau_{i}$ as a function of $\alpha$. We also calculate the signal $S_{r}\left(\Gamma_{\mathrm{LB}}, \alpha\right)$ from the applied angle $\alpha$ at the QWP and the acting $\mathrm{THz}$ field $\Gamma_{\mathrm{LB}}$ from the measurement at $45^{\circ}$ and plot it in red on a symbolic log scale [linear $x$-scale between $-3^{\circ}$ and $3^{\circ}$ continued as $-\log (\alpha)$ and $\log (\alpha)$, respectively] to resolve small angles properly. The kink at $3^{\circ}$ is where the scale changes. Note that at the maximum $\left(\tau_{2} \hat{=} \hat{\Gamma}_{\mathrm{LB}}=2.37^{\circ}\right)$, we are well within the distortion region depicted in Fig. 2 indicated by the green dashed line. To picture this, we show the single measurement at a QWP angle of $\alpha=-0.5^{\circ}$ in Fig. $4(\mathrm{~b})$ in black. This waveform is highlighted in Fig. 3(a) as a black dashed line. The red line in Fig. 4(b) shows the signal we calculate from a measurement at $45^{\circ}$ using Eq. (1). This demonstrates the case where the applied $\mathrm{THz}$ field is strong enough to flip the sign of the measured signal against the bias of the small angle.

We find excellent agreement between our experimental data and theory. The largest deviations are found at high $\Gamma_{\mathrm{LB}}$, where our setup could not be optimized further to follow the large voltage changes with acceptable stability at high measurement speeds and allow for signals differing by orders of magnitude without any change in the electronic amplification. For the minimal field, which resembles the case of an intermediate field strength and the very weak just not vanishing field, we find that the method holds.

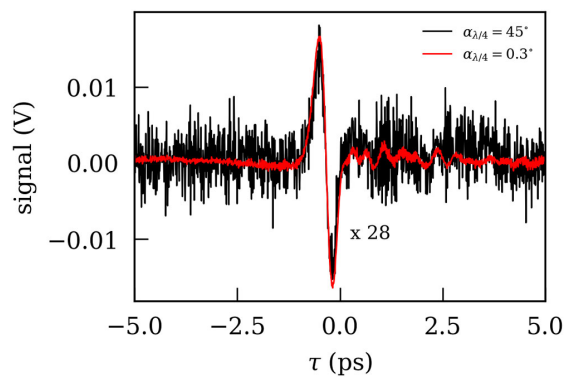

Fig. 5. Collection of measurements of a weak field in the standard method (black) compared with the amplified scheme (red). The black curve is multiplied by our amplification factor of 28 and took 6.5 times as many shots from our laser system.

For the purpose of this study and to rule out the influence of the detection system, we use the same electronic amplification for all angles inducing slight saturation features at measurements with high $\Gamma_{\mathrm{LB}}$ and small angles of $\alpha$. Optimizing electronics for certain measurement conditions improves the sensitivity beyond what is presented here.

The $\mathrm{THz}$ pulses investigated in this study were generated from $800 \mathrm{~nm}$ pulses of $37.5 \mu \mathrm{J}$. To significantly reduce $\Gamma_{\mathrm{LB}}$, we reduced the pump intensity $P_{G}$ incident on the generation crystal to $0.165 \mu \mathrm{J}$, which corresponds to a reduction of 200 of the maximum of the $\mathrm{THz}$ field. The corresponding field strength then is $\Gamma_{\mathrm{LB}}=0.045^{\circ}$. We measured the $\mathrm{THz}$ field at the standard configuration of $\alpha=45^{\circ}$ (black) and at $\alpha=0.3^{\circ}$. From Fig. 2, we read an amplification factor of 28. In Fig. 5, we show the measurement at $45^{\circ}$ times the amplification of 28 in black and the measurement at $\alpha=0.3^{\circ}$ in red. While the black line is the collection of about 15 min integration time $\left(8.7 \cdot 10^{5}\right.$ shots $)$, the measurement at the amplified scheme took only $133 \mathrm{~s}\left(1.3 \cdot 10^{5}\right.$ shots). This amplification factor of 28 and the reduced measurement time of a factor of 6.5 demonstrates the improvement at measuring comparably weak $\mathrm{THz}$ pulses. The THz fields at the crystal are of the order of $<5 \mathrm{kV} / \mathrm{cm}^{-1}$, following Ref. [11]. The measurements of field strengths of a few degrees presented here correspond roughly to $1 \mathrm{kV} / \mathrm{cm}^{-1}$ per degree of induced birefringence.

The extinction ratio of the detection setup becomes the main concern when aiming at detecting weak $\mathrm{THz}$ fields. Linear polarizers can have extinction ratios greater than $10^{9}$. The WP (as seen in Fig. 1) that separates the two polarizations has an extinction on the order of $10^{5}$. We observed that the QWP nearly does not affect extinction, but that the somehow reduced extinction ratio obtained in our setup is mainly limited by the detection crystal, which limits the extinction ratio to about $10^{4}$. A better $\mathrm{ZnTe}$ crystal with better optical quality is highly desirable for the detection of even weaker THz fields. As seen by the formula for the maximum amplification, $\Lambda=\frac{1}{4 \sqrt{r}}$, a $r$ of $10^{-5}$ would give amplifications of 79, while realizing $r=10^{-6}$ would give amplifications of 250 .

We applied this amplification to the technique of $\mathrm{THz}$ emission spectroscopy, but it could also find interest in the field of $\mathrm{THz}$ absorption spectroscopy (steady state or with optical excitation), where relatively thick emitting crystals are used in the generation. Thick $\mathrm{THz}$ crystals increase the $\mathrm{THz}$ maximum 
field emitted while reducing its bandwidth, due to the group velocity mismatch between the $\mathrm{THz}$ and the input visible or near-infrared pulse. Combining our technique with the use of thinner emitting crystal could increase the bandwidth detection in $\mathrm{THz}$ absorption setups.

Second, in THz spectroscopy of materials in the gas phase, the rotational free induction decay (FID) usually lasts tens of picoseconds (corresponding to absorption lines of tens of $\mathrm{GHz}$ ) and is usually orders of magnitude weaker than the input field. EOS detection of gas phase FID can be strongly amplified without distortion while the distorted input field can be ignored for analysis [12]. This could increase significantly the detectivity of such setups.

$\mathrm{THz}$ spectroscopy is also very popular when used with a high repetition rate system, at MHz frequencies. When EOS is used and when the gating light is in large excess to saturate the detector, our technique can be used. The $\mathrm{THz}$ fields at $\mathrm{MHz}$ repetition rate are typically weak and far from the limit where detection-induced distortions appear.

\section{CONCLUSION AND OUTLOOK}

We present a concise understanding of the possible amplification in the EOP of $\mathrm{THz}$ pulses by biasing the gating field polarization state. We deduce boundaries within which it is possible to use this amplification technique with a single measurement with negligible distortions. We demonstrate this at a comparably weak field, which is nearly impossible to detect in the standard method. With little additions to the typical detection scheme, sensitivities can strongly be enhanced in a variety of applications not limited to $\mathrm{THz}$ emission experiments [13,14], but also in optical pump $\mathrm{THz}$ probe experiments significantly reducing measurement time $[15,16]$. Making these changes to the typical EOP allows for the measurement of weak fields such as those generated in chemical and photophysical processes. It also helps in fiber-coupled detection schemes [17]. The requirements for this application are efficient optics in terms of polarization and the availability of excess gating photons while staying below the damage thresholds of the detection crystal and the optical detector, respectively.

\section{APPENDIX A: MUELLER FORMALISM}

By using Stokes vector and Mueller matrices, we derive Eq. (1). The two full matrices for the optical system for each of the balanced detectors consist of the following:

- a linear polarizer at $0^{\circ}$ with extinction ratio $r: \mathrm{LP}(0, r)$,

- the $\mathrm{ZnTe}$ crystal, which acts as a variable wave plate at $45^{\circ}$ : $\mathrm{EO}\left(\Gamma_{\mathrm{LB}}\right)$

- a QWP at angle alpha: $\operatorname{QWP}(\alpha)$,

- a linear polarizer for the two PDs of the balanced detector PD1: $\operatorname{LP}(0, r)$ and for $\mathrm{PD} 2: \operatorname{LP}\left(\frac{\pi}{2}, r\right)$ at $0^{\circ}$ and $90^{\circ}$, both with extinction ratio $r$.

We use two scalars, $\mathrm{A} 1$ and $\mathrm{A} 2$, to account for the two attenuators in Fig. 1 and ensure balancing in the very end.

The input Stokes $S_{\text {in }}$ vector corresponds to unpolarized light with an intensity depending on the first attenuator $A_{1}$. Without
$\mathrm{A}_{1}$, we ensure an intensity of 1 on each PD with $\alpha=45^{\circ}$, in absence of a THz field $\left(\Gamma_{\mathrm{LB}}=0\right)$ by setting $S_{\mathrm{in}}=\left(\begin{array}{l}4 \\ 0 \\ 0 \\ 0\end{array}\right)$.

The intensity on PD1 is the first element (A1) of the Stokes vector corresponding to the intensity at PD1 without attenuator,

$$
\begin{aligned}
I_{\mathrm{PD} 1}\left(\Gamma_{\mathrm{LB}}, \alpha, r\right)= & \left(\mathrm{LP}\left(\frac{\pi}{2}, r\right) \cdot \mathrm{QWP}(\alpha) \cdot \mathrm{EO}\left(\Gamma_{\mathrm{LB}}\right)\right. \\
& \left.\cdot \mathrm{LP}(0, r) \cdot S_{\text {in }}\right),
\end{aligned}
$$

whereas the intensity without attenuator on PD2 is

$$
\begin{aligned}
I_{\mathrm{PD} 2}\left(\Gamma_{\mathrm{LB}}, \alpha, r\right)= & \left(\mathrm{LP}(0, r) \cdot \operatorname{QWP}(\alpha) \cdot \mathrm{EO}\left(\Gamma_{\mathrm{LB}}\right)\right. \\
& \left.\cdot \mathrm{LP}(0, r) \cdot S_{\mathrm{in}}\right) .
\end{aligned}
$$

The difference between these two intensities is the last polarizer, which is applied in the formalism and corresponds to the two outputs of the WP. Finally, considering the attenuation, the detected signal calculated with Eq. (1) is the difference between these intensities,

$$
S_{r}\left(\alpha, \Gamma_{\mathrm{LB}}\right)=\mathrm{A} 1 \times\left(I_{\mathrm{PD} 1}-\mathrm{A} 2 \times I_{\mathrm{PD} 2}\right),
$$

with $\mathrm{A} 1=\frac{1}{I_{\mathrm{PD} 1}(0, \alpha, r)}$ and $\mathrm{A} 2=\frac{I_{\mathrm{PD} 1}(0, \alpha, r)}{I_{\mathrm{PD} 2}(0, \alpha, r)}$ to ensure balancing of the PDs at $\left(\Gamma_{\mathrm{LB}}=0\right)$.

The Mueller matrices used above are

$$
\begin{aligned}
\operatorname{LP}(0, r) & =\frac{1}{2}\left(\begin{array}{cccc}
(1+r) & (1-r) & 0 & 0 \\
(1-r) & (1+r) & 0 & 0 \\
0 & 0 & 0 & 0 \\
0 & 0 & 0 & 0
\end{array}\right), \\
\operatorname{LP}\left(\frac{\pi}{2}, r\right) & =\frac{1}{2}\left(\begin{array}{cccc}
(1+r) & (r-1) & 0 & 0 \\
(r-1) & (1+r) & 0 & 0 \\
0 & 0 & 0 & 0 \\
0 & 0 & 0 & 0
\end{array}\right), \\
\operatorname{QWP}(\alpha) & =\left(\begin{array}{cccc}
1 & 0 & 0 & 0 \\
0 & \cos (2 \alpha)^{2} & \frac{1}{2} \sin (4 \alpha) & -\sin (2 \alpha) \\
0 & \frac{1}{2} \sin (4 \alpha) & \sin (2 \alpha)^{2} & \cos (2 \alpha) \\
0 & \sin (2 \alpha) & -\cos (2 \alpha) & 0
\end{array}\right),
\end{aligned}
$$

and

$$
\mathrm{EO}\left(\Gamma_{\mathrm{LB}}\right)=\left(\begin{array}{cccc}
1 & 0 & 0 & 0 \\
0 & \cos \left(\Gamma_{\mathrm{LB}}\right) & 0 & \sin \left(\Gamma_{\mathrm{LB}}\right) \\
0 & 0 & 1 & 0 \\
0 & -\sin \left(\Gamma_{\mathrm{LB}}\right) & 0 & \cos \left(\Gamma_{\mathrm{LB}}\right)
\end{array}\right)
$$

Funding. European Research Council (714586); Schweizerischer Nationalfonds zur Förderung der Wissenschaftlichen Forschung (NCCR-MUST).

Acknowledgment. We thank Prof. Thomas Feurer (University of Bern) for fruitful discussions. 
Disclosures. The authors declare no conflict of interest.

\section{REFERENCES}

1. G. Gallot and D. Grischkowsky, "Electro-optic detection of terahertz radiation," J. Opt. Soc. Am. B 16, 1204-1212 (1999).

2. C. Riek, D. V. Seletskiy, and A. Leitenstorfer, "Femtosecond measurements of electric fields: from classical amplitudes to quantum fluctuations," Eur. J. Phys. 38, 024003 (2017).

3. F. D. J. Brunner, J. A. Johnson, S. Grübel, A. Ferrer, S. L. Johnson, and T. Feurer, "Distortion-free enhancement of terahertz signals measured by electro-optic sampling. I. Theory," J. Opt. Soc. Am. B 31, 904-910 (2014).

4. J. Degert, M. Cornet, E. Abraham, and E. Freysz, "Simple and distortion-free optical sampling of terahertz pulses via heterodyne detection schemes," J. Opt. Soc. Am. B 33, 2045-2050 (2016).

5. J. A. Johnson, F. D. J. Brunner, S. Grübel, A. Ferrer, S. L. Johnson, and T. Feurer, "Distortion-free enhancement of terahertz signals measured by electro-optic sampling. II. Experiment," J. Opt. Soc. Am. B 31, 1035-1040 (2014).

6. S. Ahmed, J. Savolainen, and P. Hamm, "Detectivity enhancement in THz electrooptical sampling," Rev. Sci. Instrum. 85, 013114 (2014).

7. O. Kliebisch, D. C. Heinecke, and T. Dekorsy, "Ultrafast time-domain spectroscopy system using $10 \mathrm{GHz}$ asynchronous optical sampling with $100 \mathrm{kHz}$ scan rate," Opt. Express 24, 29930-29940 (2016).

8. J. Helbing and P. Hamm, "Compact implementation of Fourier transform two-dimensional IR spectroscopy without phase ambiguity," J. Opt. Soc. Am. B 28, 171-178 (2011).

9. Q. Wu, T. D. Hewitt, and X. C. Zhang, "Two-dimensional electro-optic imaging of THz beams,” Appl. Phys. Lett. 69, 1026-1028 (1996).
10. S. Namba, "Electro-optical effect of zincblende," J. Opt. Soc. Am. 51, 76-79 (1961).

11. T. Löffler, T. Hahn, M. Thomson, F. Jacob, and H. G. Roskos, "Large-area electro-optic ZnTe terahertz emitters," Opt. Express 13, 5353-5362 (2005).

12. M. B. Agranat, I. V. I'ina, and D. S. Sitnikov, "Application of terahertz spectroscopy for remote express analysis of gases," High Temp. 55, 922-934 (2017).

13. C. S. Ponseca, A. Arlauskas, H. Yu, F. Wang, I. Nevinskas, E. Dūda, V. Vaičaitis, J. Eriksson, J. Bergqvist, X.-K. Liu, M. Kemerink, A. N. Krotkus, O. Inganas, and F. Gao, "Pulsed terahertz emission from solution-processed lead iodide perovskite films," ACS Photon. 6, 1175-1181 (2019).

14. B. Guzelturk, R. A. Belisle, M. D. Smith, K. Bruening, R. Prasanna, Y. Yuan, V. Gopalan, C. J. Tassone, H. I. Karunadasa, M. D. McGehee, and A. M. Lindenberg, "Terahertz emission from hybrid perovskites driven by ultrafast charge separation and strong electron-phonon coupling," Adv. Mater. 30, 1704737 (2018).

15. P. Krauspe, D. Tsokkou, M. Causa, E. Buchaca-Domingo, Z. Fei, M. Heeney, N. Stingelin, and N. Banerji, "Terahertz short-range mobilities in neat and intermixed regions of polymer:fullerene blends with controlled phase morphology," J. Mater. Chem. A 6, 22301-22309 (2018).

16. C. S. Ponseca, A. Yartsev, E. Wang, M. R. Andersson, D. Vithanage, and V. Sundström, "Ultrafast terahertz photoconductivity of bulk heterojunction materials reveals high carrier mobility up to nanosecond time scale," J. Am. Chem. Soc. 134, 11836-11839 (2012).

17. C. Szwaj, C. Evain, M. L. Parquier, P. Roy, L. Manceron, J.-B. Brubach, M.-A. Tordeux, and S. Bielawski, "High sensitivity photonic time-stretch electro-optic sampling of terahertz pulses," Rev. Sci. Instrum. 87, 103111 (2016). 